patient. In contrast, support from management was one aspect of context which assisted with facilitation efforts.

Conclusions In addition to managerial support, establishing a team of practitioners to lead facilitation of the CSNAT intervention and regularly review implementation progress, is vital for implementation success.

This study was funded by Dimbleby Cancer Care.

\section{0-4 ENABLING SUCCESSFUL HOSPITAL DISCHARGE TO HOME AT END-OF-LIFE: HOW CAN WE SUPPORT FAMILY CARERS?}

${ }^{1}$ Gail Ewing, ${ }^{2}$ Lynn Austin, ${ }^{2}$ Debra Gibson, ${ }^{2}$ Gunn Grande. ${ }^{1}$ University of Cambridge, Cambridge, UK; ${ }^{2}$ University of Manchester, Manchester, UK

\subsection{6/bmjspcare-2016-001245.4}

Background Successful hospital discharge and prevention of readmission often depend on carers' ability to support patients.

Aim To investigate how carers are supported during patient discharge from acute care towards end-of-life (EOL) and suitability of using the Carer Support Needs Assessment Tool (CSNAT) to improve carer support at discharge.

Methods Qualitative design: focus groups (FGs) with 40 practitioners supporting patient discharge from three English acute hospital trusts; interviews with 22 carers of patients discharged. 14 practitioners and five carers joined two final workshops. FGs/ interviews/workshops explored current discharge processes and potential value of using CSNAT. Thematic framework analysis conducted.

Results Discharge processes were heavily focussed on patients' needs: there was no systematic approach to supporting carers. Practitioners and carers viewed CSNAT as highly relevant and could be used to facilitate much needed EOL conversations which often were absent and to manage carers' expectations of their caregiving role at EOL, including support available (or not) in the community. They also provided advice on feasibility of using the five stage CSNAT approach at discharge.

- Stage 1. CSNAT introduction was seen as crucial, to overcome carer reluctance for support for themselves and to avoid it being viewed as 'another leaflet'

- Stage 2. Carers' consideration of needs: useful to help manage expectations of caregiving, but carers need to be given time to reflect

- Stage 3. Assessment conversation: CSNAT questions seen as a useful trigger, but a separate space and a separate focus from patents needed.

- Stage 4. Action planning: an essential part of the process giving out the CSNAT was not 'job done'

- Stage 5. Review: challenge in this context is the transition to home, but CSNAT as a carer-held record was a possible solution.

Conclusion CSNAT shows good potential to enhance carer support at hospital discharge and play a role in preventing readmissions towards EOL.

Funder: Marie Curie.

\section{Conference Papers 2}

\section{0-5 COMMUNICATION ABOUT CARDIOPULMONARY RESUSCITATION DECISIONS AT A UK HOSPICE INPATIENT UNIT}

Joanna Davies, Pauline Dand. Pilgrims Hospices, Canterbury, UK

10.1136/bmjspcare-2016-001245.5

Background A 2014 court ruling in the UK established that the only justification for NOT discussing a "Do Not Attempt Cardiopulmonary Resuscitation"(DNACPR) order with a competent patient is either patient choice or potential "harm" to the patient (not distress).

Aims This study aimed to establish current practice in communication when making DNACPR decisions, the impact of the ruling and the interpretation of "harm".

Methods The records of 150 hospice inpatients admitted after the ruling were screened. An anonymous survey was sent to hospice doctors and hospice nurses trained to complete DNACPR orders.

Results DNACPR decisions were made without discussion with competent patients in $6 / 150$ cases. Reasons documented included: patient choice, the decision was implied from previous discussions, the patient was too unwell. All six decisions were discussed with the family.

Survey response rate was 90\% (28/31) with equal numbers of specialist nurses and doctors. $21 / 28$ respondents made DNACPR decisions at least monthly, 6/28 had made these decisions without discussion with a competent patient in the past six months, 20/28 were aware of the ruling and 16/28 felt it would impact on their practice.

Examples of impact on practice included; increased awareness of need to keep up-to-date, forcing earlier DNACPR discussions, pressure to discuss decisions with all patients, increased likelihood of exploring patient choice, increased involvement of family. Interpretations of "harm" included: more than distress, physical harm to self/others, psychiatric disorder, damage to doctor-patient relationship, distress close to the end-of-life.

Conclusions Only a minority of decisions were not discussed with competent patients. Not all relevant health care professionals are aware of the recent court ruling. Of those who were, over half felt it would impact upon their communication practice. There is a need for clarification of what constitutes harm rather than distress.

\section{0-6 DELIVERING INTEGRATED HOSPICE BASED CARE IN MOTOR NEURONE DISEASE}

${ }^{1,2}$ Claire Ferguson, 'Suzanne McArthur, ${ }^{1,2}$ Nikki Reed. ${ }^{1}$ Marie Curie Hospice, West Midlands, Solihull, UK; ${ }^{2}$ Heart of England NHS Foundation Trust

\subsection{6/bmjspcare-2016-001245.6}

Background 2016 NICE guidelines on assessment and management of Motor Neurone Disease (MND) recommend that patients should have access to multidisciplinary, integrated care with access to local services and support groups. 